\title{
Dual Parallel Sequence Progression as Vascular Pseudo-Reactivities to Tumor Necrosis and to Proliferation of Glioblastoma Cells
}

\section{Lawrence M Agius}

Department of Pathology, Mater Dei Hospital, University of Malta Medical School, Msida, Malta, Europe

*Corresponding author: Lawrence M Agius, Department of Pathology, Mater Dei Hospital, University of Malta Medical School, Msida, Malta, Europe, Tel: 356-21451752; E-mail: lawrence.agius@um.edu.mt

Received date: November 04, 2016; Accepted date: November 21, 2016; Published date: November 25, 2016

Copyright: (c) 2016 Agius LM. This is an open-access article distributed under the terms of the Creative Commons Attribution License, which permits unrestricted use, distribution, and reproduction in any medium, provided the original author and source are credited.

\begin{abstract}
Directional projections of glioblastoma biology include the constitutive clonality of a lesion that infiltrates in terms of its established potential proliferation involving predominantly poorly differentiated cell populations. In large measure, inclusive parameters correlate with the presence of contrastingly different types of genetic lesion, such as for example, the implication of p53 mutation versus the amplification/over-expression of the Epidermal Growth Factor Receptor (EGFR) in the neoplastic cells. Involvement of vasculature and of intense vascularization of multiple foci of the evolving glioblastoma emphasizes the distinctive link to multiple foci of tumor necrosis. Collaborative features include also an apparent propensity for pseudo-multifocality arising from processes of highly active foci of infiltration within the white matter. Cancer stem cells are believed to be the propagating cell component in gliomas and may differ from glioma initiating cells responsible for the establishment and survival of the neoplasm. Therapeutic resistance may specifically relate to resistance of the glioma stem cells that are found in small numbers in the tumor. Patient age is a distinctive feature of glioblastoma that progresses in close parallel with the vasculogenesis in neoplastic cell infiltration of adjacent tracts such as the corpus callosum and also the cerebral white matter of one or both cerebral hemispheres.
\end{abstract}

Keywords: Glioblastoma; Angiogenic factor; Oncogenic pathways; Hyperplasia

\section{Introduction}

Glioblastoma is characterized by prominent microvascularity and by a strong expression of proangiogenic cytokines [1], potentiating the value of targeting angiogenesis. Vascular endothelial growth factor (VEGF) signaling through VEGFR-2 is the central factor inducing angiogenesis in glioblastoma [2]. System exclusion of options in the lesions of the genetic pathways controlling and modulating generation and progression of glioblastomas indicate a realization of the heterogeneous potentiality for malignant transformation. Multimodal imaging is critical in assessing edema, tumor vascularity and cell proliferation in recurrent glioblastoma [3], but is not so useful in detecting tumor cell infiltration. Emergence of variants of glioblastoma implicates age of the patient that encodes the processes promoting interactivities of various cells of origin in glioblastoma genesis. The angiogenic factor YKL-40 (CHI3L1) targets glioblastoma-stem like cells to drive angiogenesis and vasculogenic mimicry [4]. Participation of individualistic options suggests the possible alternative convergence of dual or possibly multiple components in progression of high-grade astrocytomas; this is in view of expanding glioblastomas that infiltrate particularly the subcortical regions of the hemispheres.

Glioblastomas are highly heterogeneous lesions in terms of both molecular and other genetic markers, including also epigenetic factors and developmental factors. The pro-permeability factor Semaphorin $3 \mathrm{~A}$ produced by the tumor is related to loss of endothelial barrier integrity [5]. The molecular diversity of brain tumors highlights links between the coagulome and oncogenic pathways; oncogenes (EGFR, MET) and tumor suppressors (PTEN, TP53) may affect release of tissue factor [6].
Spinal glioblastoma patients have a better outcome after surgery and subsequent post-surgical radiotherapy and/or chemotherapy than others who receive surgery only [7]; such post-operative treatment may include inhibitors of receptor tyrosine kinases. The glioblastoma has been redefined as specificity of both glioma stem cells/progenitor cells in astrocytic malignant transformation. Galectin-3 is a betagalactoside binding protein and is a key factor in glioblastoma growth under hypoxic and nutrient-deprived conditions [8],

The HOX cluster group is implicated in gliomagenesis and illustrates the close parallel progression of neogenesis of tumors and normal developmental pathways. In view of the collaborative factors in glioblastoma spread, there would potentially arise an important role for various histopathologic and biologic features of glioblastoma that conform to the emergence of infiltrative behavior.

CD93 is a central regulator of glioma angiogenesis, and acts on cytoskeletal elements for cell-cell and cell-matrix adhesion, and Dexamethasone influences considerably immunologic interference in recurrent glioblastoma $[9,10]$. Current preclinical research focuses on predictive biomarkers and mechanisms of tumor/vascular resistance [11]. Inefficient anti-VEGFR administration still limits therapeutic efficacy [12].

The highly heterogeneous nature of a given glioblastoma would include the processes of tumor genesis as further compounded by necrosis and hyperplasia of regional vessels. Oct-3/4, a self-renewal regulator in stem cells may permit glioblastoma cells to adapt to lowoxygen environments [13]. CD90 interacts with integrins expressed by tumor-associated macrophages and is present on endothelial cells, fibroblasts and pericytes of glioblastoma vessels [14]. Hypoxia enhances tumor aggressiveness and negatively correlates with a decrease in patient outcome [15]. 


\section{Constitutional Attributes}

Constitutive re-coupling of various entity components belies the usual conceptual frameworks of glioblastoma genesis as generation of undifferentiated progenitor glioma cells that generally react only weakly with glial fibrillary acidic protein immunochemistry.

Multi-regional imaging analysis more effectively characterizes glioblastoma heterogeneity in terms of prognostic biomarkers [16]. The expression of the CXCR4 receptor and its ligand stromal derived factor 1 correlates with cancer cell migration, tumor angiogenesis and recruitment of vascular progenitor cells, with maintenance of the perivascular stem cell niche [17]. The pSTAT3-VEGF signaling pathway may prove to be a regulatory mechanism during peritumoral edema formation and glioblastoma angiogenic progression [18]. Glioblastoma adaptation to bevacizumab therapy, with uncoupling of glycolysis from oxidative phosphorylation represents an adaptive response to therapy [19].

The highly heterogeneous biologic nature of glioblastoma correlates with the widespread infiltration of the white matter of the cerebral hemisphere or hemispheres. The oncogene astrocyte elevated gene-1 activates multiple signaling pathways that drive invasion and angiogenesis by interaction with Akt2 in glioblastoma [20]. Such implicated biologic processes include changes in cell cycle progression, receptor amplification or constitutive activation, anti-apoptosis, cell motility and proliferation. DNA-dependent protein kinase catalytic subunit suppression in glioblastoma cells partly reduced ionizing radiation-inflicted migration, invasion and tube formation of human microvascular endothelial cells [21].

Also implicated are a multitude of signaling pathways that involve roles for the retinoblastoma gene and the $\mathrm{p} 53$ protein.

It is within the contextual modifying influences of the initial malignant transformation steps that glioblastoma proves an end-result of multiple modulations primarily affecting receptor tyrosine kinases involving in particular the epidermal growth factor receptor, and platelet derived growth factor receptor that act on downstream effectors such as phosphatidylinositol 3-kinase, mitogen-activated protein kinase and signal transducer and activator of transcription. Hypoxia enhances aggressive tumor attributes but correlates negatively with a decrease in outcome [22]. Fatty acid uptake and lipid storage are enhanced by hypoxia-inducible factor 1 alpha and may contribute to tumor cell growth after hypoxia-reoxygenation [23].

Constitutive re-composition of the neoplasm further confirms the intensive dynamics of the unique parameters of glioblastoma origin and progression. Susceptibility weighted imaging shows tumor vasculature and microhemorrhages beyond the use of conventional MRI [24]. Compromised barrier function in tumor vessels may possibly permit communication between tumor microenvironment and peripheral blood [25].

It is beyond the transformation of the individual malignant astrocyte that there develops corroborative intensification of the glomeruloid vessel types as strictly regional tissue and microenviornmental involvement in early tumorigenesis.

\section{Comparative Phenomena}

Comparative coordinates of a space-time dimensionality conclusively re-define the extreme injury to cell cycling that evolves with the rapidly progressive nature of the ischemia within the neoplasm. Glioblastoma critically depends on growth of new endothelial vasculature [26]. The correlates of such glomeruloid vessels within the central regions of tumor necrosis or with the advancing infiltrative processes indicate specificity towards responsive hypoxic events in differential tumor evolution.

The recognition of vasculogenic mimicry has led to the realization of a tumor-cell-constituted meshwork that is independent of endothelial cells and that correlates with poor prognosis [27].

Divergence can be viewed as a composite end-product of the highly converging influences of glioblastoma progression. Transcriptionally active 06-methyguanine-DNA-methyltransferase effectively removes alkyl adducts and prevents thus cross-linking and resistance to alkylating drugs [28]. Oxygenation levels in particular are critical to tumor heterogeneity and compartmentalization [29].

Divergence and alternating convergence phenomena cooperatively instigate the modulatory dimensions of ischemia as glomeruloid vasculogenesis within the formative influences of an integral lesion that proves histopathologically highly heterogeneous.

Constitutive contrasting phenomena attest to infiltrative behavior of a lesion beyond simple biologic parameters of cell cycling. Chronic arterial hypertension may impede growth of glioblastomas through impaired vasoreactivity, disturbed perfusion and induced hypoxia [30]. In measured compromise of ischemia and cell proliferation, there arises the growth of a tumor that is largely composed of the widely spreading groups or sometimes ribbon morphologies of a highly malignant neoplasm. Flavonoids have an anti-cancer effect and appear to induce caspase-dependent activation of both the extrinsic and intrinsic apoptotic pathways [31]. Hypoxia inducible factor 1 alpha plays a crucial role in the malignant phenotypes of glioblastoma [32]. Such contrasting phenomena are particularly significant in view of the emergence of progressively de-differentiating parameters affecting many of the neoplastic cells. Suicide gene therapy involving adult stem cells could effectively eliminate glioblastoma cells in vivo [33].

In collaborative interventions at interactivity there develops a dynamic disequilibrium that is largely dominated by widely infiltrating neoplastic cells that collaborate, in turn, with extensive, prominent foci of tumor necrosis.

\section{Parallelism}

The parameters of inclusive dimensionality convert a pseudoregional series of pathways towards the global pathology of a hemisphere that includes cerebral edema.

In parallel with the establishment of the ongoing constitutive nature of the glioblastoma, there is an inherent propensity for tumor regenerative abilities that redefine substrate heterogeneity. MicroRNA-21 is an oncogenic miRNA that can be inhibited by benzamides [34]. The significance of performance dynamics of the infiltrative process includes re-constitutive potential for genesis and infiltration. Antiangiogenic therapy has focused on inhibitors of the vascular endothelial growth factor signaling pathway [35].

Indices of spectrum manifestation are parameters of progression in a highly developed hierarchy in the emergence of the infiltrative processes. Intracranial xenograft glioblastoma in mouse models treated with uncomplexed miR-148a and miR-31 antagomirs showed reduced proliferation, stem cell depletion, and normalized tumor vasculature [36]. 
The transforming nature of the malignant process incorporates the substrate formulations of a tumor type that is conformationally and constitutively executed.

Disproportionate contrast enhancement, relative to biologic attributes of the tumor cell, correlates with integrative processes of lesion promotion and progression. It is within such proposed conditioned neoplastic formulation that constitutive divergence emerges as infiltrative tumor growth. Small interfering RNA loaded nanoparticles do not induce cytotoxicity in glioblastoma cells but markedly inhibit cell proliferation [37].

The 'butterfly' lesion is a primary reconstitution of a tumor type that propagates as infiltrative phenomena of heterogeneous type.

Accompanying foci of tumor necrosis involve viable neoplastic cells that form pseudo-palisades around necrotic foci that are of a dynamic character.

The aberrant progression of a given glioblastoma promotes the propensity for tumor progression within multifocal regions of hemispheric involvement as seen within the white matter; the perivascular and subpial containment influences dynamics of infiltration and biologic proliferative activity of the neoplastic elements.

\section{Sequentiality of Tumor Progression}

A sequence disorder in genetic lesion creation appears to operate in glioblastoma development, as evidenced also by the observed exclusion of p53 with EGF receptivity, and in the mutational or ampllfication/ overexpression of genes that in turn correlate with patient age and with primary versus secondary status of glioblastomas. The relative associations of $p 53$ gene mutation and of $\mathrm{p} 53$ protein accumulation in the cytoplasm of tumor cells induce the phenomenon of secondary glioblastoma, in the younger patient, that is in patients with a median age of 45 years. The primary glioblastoma, occurring mostly in patients over 65 years of age, would allow for a putatively permissive emergence of a lesion that is related in most instances to EGFR amplification and over-expression.

The multiplicity of ongoing genetic injuries is an accumulative process of mutations and over-expression that are necessarily global forces of inducing modulation of proliferative and clonal progression. $\mathrm{N}$-myc downstream-regulated gene 2 is implicated in glioblastoma with developmental involvement in astrocytic proliferation, differentiation, transmembrane transport, and stress response [38].

It is highly significant that clonality is manifestly present not only in the usual glioblastoma, but is reflected also in vascular endothelial cell hyperplastic foci, and also in gliosarcomas. Such features corroborate with the prolongation of action of a putative global multilineage injurious lesion in transformation to a malignant phenotype. Markers of systemic inflammation such as neutrophil-to-lymphocyte ratios correlate with prognostic outcome in patients with glioblastoma [39].

\section{Profiles of Oncogenesis}

Performance profiles of constitutively activated receptors such as the vIII variant of the EGFR are particularly significant as ongoing dynamics of the initial malignant transformation process inducing gliomagenesis.

The particular profile formulations of progression of a glioblastoma lesion become established within a very early stage of the malignant lesion emergence and would implicate clonality studies as, for example, the biologic implications of $\mathrm{p} 53$ mutation rates.

The pseudo-encapsulation as seen in the giant cell variant of glioblastoma correlates with a lesser ability to infiltrate the cerebral white matter accompanied by contextually less proliferative activity of the giant tumor cell lesion. Conditioning parameters in various types of glioblastoma progression may be linked to the constitutive receptor activation. Also, human cytomegalovirus proteins and nucleic acids may act as glioma-associated target for anti-CMV agents in many cases of glioblastoma [40].

The intrinsic nature of malignant transformation may target, for example, the p53 mutations or induce p53 protein accumulation. Corroborative partner contrast formulations are produced as highly established cell cycling dynamics and as performance attributes, as well illustrated by the secondary structures of Scherer. Glioblastoma cells are resistant to apoptosis with consequent ineffectiveness of the usual chemotherapeutic modalities [40].

\section{Concluding Remarks}

Parameter stabilization is an overall and global array of constitutive profiles that enable the progression of a highly heterogeneous lesion as glioblastoma, within contextual neoplastic genesis showing stereotyped progression. It is the actual emergence of the initial transforming process of tumorogenesis that contrasts with genetic mutations in nonmalignant but highly hyperplastic foci of endothelial cells in the glomeruloid vessels that often surround necrotic foci.

The included observation of hyperplastic vessels within the highly proliferative margins of the glioblastoma lesion further collaborates with the phenomena of coupled tumor-cell proliferation and high degrees of potential vascular progression. Conclusive parametric reconstitution of glioblastoma biology portrays the dynamics of a lesion that proliferates in terms of the established vasculogenic potential of such tumors to infiltrate the cerebral white matter.

\section{References}

1. Batchelor TT, Reardon DA, de Groot JF, Wick W, Weller M (2014) Antiangiogenic therapy for glioblastoma: current status and future prospects. Clin Cancer Res 20: 5612-5619.

2. Schiff D, Kesari S, de Groot J, Mikkelsen T, Drappatz J, et al. (2015) Phase 2 study of CT-322, a targeted biologic inhibitor of VEGFR-2 based on a domain of human fibronectin, in recurrent glioblastoma. Invest New Drugs 33: 247-253.

3. Corroyer-Dulmont A, Peres EA, Gerault AN, Savina A, Bouquet F, et al. (2015) "Multimodal imaging based on MRI and PET reveals [18F]FLT PET as a specific and early indicator of treatment efficacy in a preclinical model of recurrent glioblastoma" Eur J Nucl Med Mol imaging 42: 682-694.

4. Shao R, Taylor SL, Oh DS, Schwartz LM (2015) Vascular heterogeneity and targeting: the role of YKL-40 in glioblastoma vascularization. Oncotarget 6: 40507-40518.

5. Treps L, Edmond S, Harford-Wright E, Galan-Moya EM, Schmitt A, et al. (2015) Extracellular vesicle-transported Semaphorin3A promotes vascular permeability in glioblastoma. Oncogene 35: 3615-2623.

6. D'Asti E, Fang Y, Rak J (2014) Brain neoplasms and coagulation-lessons from heterogeneity. Rambam Maimonides Med J 5: e0030.

7. Konar SK, Maitl TK, Bir SC, Kalakoti P, Bollam P, et al. (2015) Predictive factors determining the overall outcome of primary spinal glioblastoma multiforme: an integrative survival analysis. World Neurosurg 86 : 341-348. 
8. Langenkamp E, Zhang L, Lugano R, Huang H, Elhassan TE, et al. (2015) Elevated expression of the C-type Lectin CD93 in the glioblastoma vasculature regulates cytoskeletal rearrangements that enhance vessel function and reduce host survival. Cancer Res 75: 4504-4516.

9. Ellsworth S, Grossman SA (2015) Comment on 'Dexamethasone exerts profound immunologic interference on treatment efficacy for recurrent glioblastoma' Br J Cancer 113: 1632-1633.

10. Wong ET, Swanson KD (2015) Comment on 'Dexamethasone exerts profound immunologic interference on treatment efficacy for recurrent glioblastoma'. Br J Cancer 113: 1633-1634.

11. Inoue A, Tanaka J, Takahashi H, Kohno S, Ohue S, et al. (2016) Blood vessels expressing CD90 in human and rat brain tumors. Neuropathology 36: 168-180.

12. Arrillaga-Romany I, Norden AD (2014) Antiangiogenic therapies for glioblastoma. CNS Oncol 3: 349-358.

13. Goel S, Chen F, Hong H, Valdovinos HF, Hernandez R, et al. (2014) VEGF121- conjugated mesoporous silica nanoparticle: a tumor targeted drug delivery system. ACS Appl Mater Interfaces 6: 21677-21685.

14. Takahashi H, Inoue A, Kawabe Y, Hosokawa Y, Iwata S, et al. (2015) Oct-3/4 promotes tumor angiogenesis through VEGF production in glioblastoma. Brain Tumor Pathol 32: 31-40.

15. Cui Y, Tha KK, Terasaka S, Yamaguchi S, Wang J, et al. (2015) Prognostic imaging biomarkers in glioblastoma: development and independent validation on the basis of multiregion and quantitative analysis of MR images. Radiology 4:150358.

16. Richardson PJ (2016) CXCR4 and Glioblastoma. Anticancer Agents Med Chem 16: 59-74.

17. Corroyer-Dulmont A, Chakhoyan A, Collet S, Durand L, MacKenzie ET, et al. (2015) Imaging Modalities to Assess Oxygen Status in Glioblastoma. Front Med (Lausanne) 2: 57 .

18. Wang XF, Lin GS, Lin ZX, Chen YP, Chen Y, et al. (2014) Association of pSTAT3-VEGF signaling pathway with peritumoral edema in newly diagnosed glioblastoma: an immunohistochemical study. Int J Clin Exp Pathol 7: 6133-6140.

19. Fack F, Espedal H, Keunen O, Golebiewska A, Obad N, et al. (2015) Bevacizumab treatment induces metabolic adaptation toward anaerobic metabolism in glioblastomas. Acta Neuropathol 129: 115-131.

20. Hu B, Emdad L, Bacolod MD, Kegelman TP, Shen XN, et al. (2014) Astrocyte elevated gene-1 interacts with Akt isoform 2 to control glioma growth, survival, and pathogenesis. Cancer Res 74: 7321-7332.

21. Liu Y, Zhang L, Liu Y, Sun C, Zhang H, et al. (2015) DNA-PKcs deficiency inhibits glioblastoma cell-derived angiogenesis after ionizing radiation. J Cell Physiol 230: 1094-1103.

22. Di Ieva A, Lam T, Alcaide-Leon P, Bharatha A, Montanera W, et al. (2015) Magnetic resonance susceptibility weighted imaging in neurosurgery: current applications and future perspectives. J Neurosurg 24: 1-13.

23. Bensaad K, Favaro E, Lewis CA, Peck B, Lord S, et al. (2014) Fatty acid

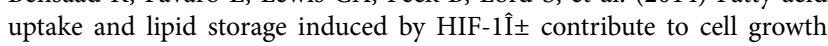
and survival after hypoxia-reoxygenation. Cell Rep 9: 349-365.

24. Harshyne LA, Nasca BJ, Kenyon LC, Andrews DW, Hooper DC (2015) Serum exosomes and cytokines promote a T-helper cell type 2 environment in the peripheral blood of glioblastoma patients" Neuro Oncol 107.

25. Gruslova A, Cavazos DA, Miller JR, Breitbart E, Cohen YC, et al. (2015) VB-111: a novel anti-vascular therapeutic for glioblastoma multiforme. J Neurooncol 124: 365-372.
26. Mao JM, Liu J, Guo G, Mao XG, Li CX (2015) Glioblastoma vasculogenic mimicry: signaling pathways progression and potential anti-angiogenesis targets. Biomark Res 3: 8.

27. Villani V, Casini B, Pace A, Prosperini L, Carapella CM, et al. (2015) The prognostic value of pyrosequencing-detected MGMT promoter hypermethylation in newly diagnosed patients with glioblastoma. Dis Markers 2015: 6047.

28. Roniotis A, Oralopoulou ME, Tzamali E, Kontopodis E, Van Cauter S, et al. (2015) A proposed paradigm shift in initializing cancer predictive models with DCE-MRI based PK parameters: a feasibility study. Cancer Inform 14: 7-18.

29. Letourneur A, Roussel S (2015) Chronic arterial hypertension impedes glioma growth: a multiparametric MRI study in the rat. Hypertens Res 38: 723-732.

30. Wang G, Wang JJ, To TS, Zhao HF, Wang J (2015) Role of SIRT1mediated mitochondrial and Akt pathways in glioblastoma cell death induced by Cotinus coggygria flavonoid nanoliposomes. Int J Nanomedicine 10: 5005-5023.

31. Nigim F, Cavanaugh J, Patel AP, Curry WT, Esaki S, et al. (2015) Targeting hypoxia-inducible factor 1 alpha in a new orthotopic model of glioblastoma recapitulating the hypoxic tumor microenvironment. J Neuropathol Exp Neurol 74: 710-722.

32. Leten C, Trekker J, Struys T, Dresselaers T, Gijsbers R, et al. (2015) Assessment of bystander killing-mediated therapy of malignant brain tumors using a multimodal imaging approach. Stem Cell Res Ther 6: 163.

33. Jiang CS, Wang XM, Zhang SQ, Meng LS, Zhu WH, et al. (2015) Discovery of 4-benzoylamino-N-(prop-2-yn-1-yl)benzamides as novel microRNA-21 inhibitors. Bioorg Med Chem 23: 6510-6519.

34. Flanigan PM, Aghi MK (2015) Adaptation to antiangiogenic therapy in neurological tumors. Cell Mol Life Sci 72: 3069-3082.

35. Wong HK, Fatimy RE, Onodera C, Wei Z, Yi M, et al. (2015) The Cancer Genome Atlas Analysis Predicts MicroRNA for Targeting Cancer Growth and Vascularization in Glioblastoma. Mol Ther 23: 1234-1247.

36. Hasanzadeh Kafshgari M, Alnakhli M, Delalat B, Apostolou S, Harding FJ, et al. (2015) Small interfering RNA delivery by polyethyleniminefunctionalised porous silicon nanoparticles. Biomater Sci 3: 1555-1565.

37. Lin K, Yin A, Yao L, Li Y (2015) N-myc downstream-regulated gene 2 in the nervous system: from expression pattern to function. Acta Biochim Biophys Sin (Shanghai) 47: 761-766.

38. Han S, Liu Y, Li Q, Li Z, Hou H, et al. (2015) Pre-treatment neutrophil-tolymphocyte ratio is associated with neutrophil and T-cell infiltration and predicts clinical outcome in patients with glioblastoma. BMC Cancer 15: 617.

39. Wakefield A, Pignata A, Ghazi A, Ashoori A, Hegde M, et al. (2015) Is CMV a target in pediatric glioblastoma? Expression of CMV proteins, pp65 and IE 1-72 and CMV nucleic acids in a cohort of pediatric glioblastoma patients. J Neurooncol 125: 307-315.

40. Dasari R, Masi M, Lisy R, Ferderin M, English LR, et al. (2015) Fungal metabolite ophiobolin $\mathrm{A}$ as a promising anti-glioma agent: in vivo evaluation, structure-activity relationship and unique pyrrolylation of primary amines. Bioorg Med Chem Lett 25: 4544-4548. 